\title{
Bend Sweep Angle and Reynolds Number Effects on Hemodynamics of S-Shaped Arteries
}

\author{
H. Niazmand and E. Rajabi Jaghargh \\ Mechanical Engineering Department, Ferdowsi University of Mashhad, Mashhad, Iran \\ (Received 2 September 2009; accepted 9 April 2010; published online 29 April 2010)
}

Associate Editor Peter E. McHugh oversaw the review of this article.

\begin{abstract}
The purpose of this study is to investigate the effects of the Reynolds number and the bend sweep angle on the blood flow patterns of S-shaped bends. The numerical simulations of steady flows in S-shaped bends with sweep angles of $45^{\circ}, 90^{\circ}$, and $135^{\circ}$ are performed at Reynolds numbers of 125,500 , and 960 . Hemodynamic characteristics such as secondary flows, vorticity, and axial velocity profiles are analyzed in detail. Flow patterns in S-shaped bends are strongly dependent on both Reynolds number and bend sweep angle, which can be categorized into three groups based on the first bend secondary flow effects on the transverse flow of the second bend. For low Reynolds numbers and any sweep angles, secondary flows in the second bend eliminate the first bend effects in the early sections of the second bend and therefore the axial velocity profile is consistent with the bend curvature, while for high Reynolds numbers depending on the bend sweep angles the secondary vortex pattern of the first bend may persist partially or totally throughout the second bend leading to a four-vortex secondary structure. Moreover, an interesting flow feature observed at the Reynolds number of 960 is that the secondary flow asymmetrical behavior occurred around the second bend exit and along the outflow straight section. This symmetry-breaking phenomenon which has not been reported in the previous studies is shown to be more pronounced in the $90^{\circ} \mathrm{S}$-shaped bend as compared to other models considered here. The probability of flow separation as one of the important flow features contributing to the onset and development of arterial wall diseases is also studied. It is observed that the second bend outer wall of gentle bends with sweep angles from $20^{\circ}$ to $30^{\circ}$ at high enough Reynolds numbers are prone to flow separation.
\end{abstract}

Keywords-S-shaped arteries, Hemodynamic, Blood flow, Secondary flow, Separation, Bend sweep angle.

Address correspondence to $\mathrm{H}$. Niazmand, Mechanical Engineering Department, Ferdowsi University of Mashhad, Mashhad, Iran. Electronic mail: hniazmand@yahoo.com, niazmand@ ferdowsi.um.ac.ir

\section{INTRODUCTION}

Atherogenesis is a complicated, very slow pathological process affected by a variety of potential risk factors, such as physiological, biochemical, genetic, and lifestyle factors, as well as hemodynamic factors. Different hypothesis regarding the mechanism of atherogenesis have been introduced in previous studies. Local hemodynamic factors are broadly accepted to play a critical role in genesis, development, and distribution of atherosclerosis. ${ }^{4,26}$ Atherosclerotic lesions can occur anywhere in arteries but usually originate at the outer wall of bifurcations and inner wall of curved arteries. This nature of atherosclerotic lesions cannot be explained by any of the above-mentioned risk factors; however, it has been shown that the hemodynamic factors can control the localization of atherosclerosis within the arteries. ${ }^{26}$ Therefore, it is important to investigate fluid dynamic factors such as velocity field, vortical structures, and pressure distribution in the susceptible sites of early lesions formation.

Previous research has shown that arterial bends and bifurcations have a significant impact on the hemodynamic factors. ${ }^{6,8,25,31,36}$ Extensive clinical and computational studies have been performed to investigate fluid dynamics in bifurcations and curved arteries. Van de Vosse et al. ${ }^{37}$ Perktold et al., ${ }^{27}$ Gijsen et al.,${ }^{11}$ and Shahcheraghi et al. ${ }^{30}$ presented numerical simulations of blood flow in curved tubes. Wall hemodynamic indicators such as wall pressure (WP), wall shear stress (WSS), and their spatial gradients (WPG and WSSG) have been investigated in curved arteries and a relationship between the predisposed sites of atherosclerosis formation and the regions of low WSS and WP or intense WPG and WSSG are proposed..$^{12,32,33}$ Rappitsch et al.,${ }^{28}$ Hong et al.,${ }^{14}$ and Wada et al. ${ }^{38}$ studied mass transfer in large single and multiple curved 
arteries in which local elevation of LDL concentration in the bend inner wall are found. The effects of stenosis in curved arteries are studied by $\mathrm{Liu}^{19}$ and Yao et al. ${ }^{40}$ They found complex disturbed flow distal to plaque that may contribute to development of current lesions or genesis of new plaques.

Most earlier work, however, consider the flow in single curved arteries, whereas vessel geometries mostly include multiple bends. Consecutive bends occur in various locations of the cardiovascular system such as aortic, coronary, and femoral arteries, and can also be employed as bypass grafts. Therefore, the hemodynamics of these arteries, which are usually referred to as S-shaped arteries or reversed curvature bends are of great practical importance. Taylor et al., ${ }^{34}$ Town et al. ${ }^{35}$ Back et al., ${ }^{1}$ Banerjee et al., ${ }^{2}$ and Hoogstraten et al. ${ }^{15}$ investigate the laminar, turbulent, pulsatile, and steady blood flows in S-shaped arteries with constant bend sweep angles and curvature ratios. It was found that the secondary flow patterns in these arteries are strongly dependent on Reynolds number. Fan et al. ${ }^{10}$ studied different bypass models and showed that the $45^{\circ} \mathrm{S}$-shaped bypass can improve the hemodynamics in the bypassed arteries reducing intimal hyperplasia along the host artery floor. Johnston and Johnston ${ }^{16}$ and Lee et al. ${ }^{20}$ numerically studied the effects of nonplanarity in S-shaped bends finding more intricate coherent structures compared to planner bends.

Despite all the work performed on the simulation of blood flow in planar S-shaped arteries, the effects of different bend sweep angles and Reynolds numbers on the flow field within these geometries are not well examined. The purpose of this study is, therefore, to better identify the effects of geometry and Reynolds number on the flow in double bend configurations chosen to model a femoral artery. In the present study, steady flow within a range of physiological Reynolds numbers in three S-shaped bends with sweep angles of $45^{\circ}, 90^{\circ}$, and $135^{\circ}$ with constant curvature ratio are considered. Effects of Reynolds number and bend sweep angle on flow field, velocity patterns, secondary flows, and vortical structures are discussed in detail.

\section{PROBLEM STATEMENT}

Three double bends geometries considered in the present study are shown in Fig. 1. The bend sweep angles in the three models are $45^{\circ}, 90^{\circ}$, and $135^{\circ}$, respectively. The reference axes for measuring the turning angles are shown for the sweep angle of $135^{\circ}$ only. In all geometries, the bend curvature radius of $R_{\mathrm{C}}=6.5 D^{2,13,15}$ ( $D$ is the artery diameter which is taken as $0.006 \mathrm{~m}^{2,13,15}$ ) is considered to resemble physiologically realistic vessels, particularly the

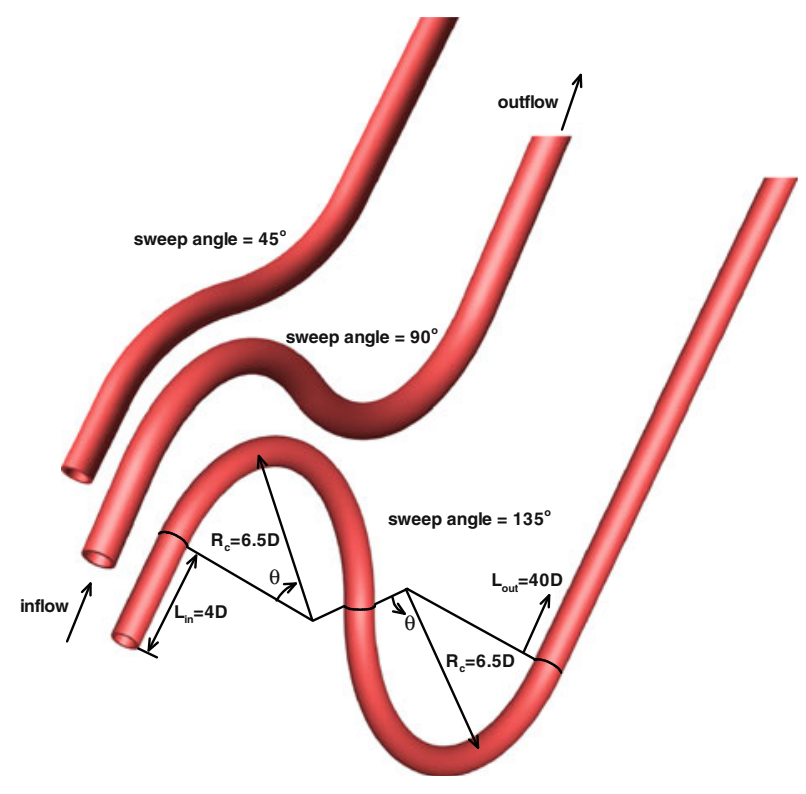

FIGURE 1. Schematic of S-shaped bends with different sweep angles and the reference axes.

femoral artery. Therefore, the curvature ratio, defined as the ratio of curvature radius to the artery radius, is equal to 13 for all cases.

As can be seen in Fig. 1, the vessel geometries consist of a rather short straight inflow region with the length of $4 D$, two identical consecutive reverse curvature bends, and a straight outflow region extended to $40 \mathrm{D}$ in order to minimize the exit boundary effects on the bends' flow field. In general, steady flows in a curved tube are characterized by the Reynolds number and geometrical parameters, such as nondimensional curvature radius and sweep angle. The steady flow calculations are performed for three physiological Reynolds numbers (based on the diameter) of 125, 500, and 960 related to the Reynolds numbers in medium to large arteries.

\section{GOVERNING EQUATIONS}

In the current study, the flow is assumed to be steady, laminar, incompressible, homogenous, and Newtonian. Blood is, in fact, a non-Newtonian shearthinning fluid; however, previous studies ${ }^{17}$ have shown that the blood non-Newtonian behavior is only of minor importance in medium to large sized arteries as is the case of present work. Furthermore, Nosovitsky et $a .^{25}$ confirmed the validity of laminar flow assumption in a stenotic $90^{\circ}$ bend by comparing the laminar flow results with those of $k-\varepsilon$ turbulence model. It was found that the effect of turbulence is small and therefore can safely be neglected. Artery wall 
is considered to be rigid, which is a reasonable assumption in medium- to large-sized arteries where the arterial wall deformability is generally taught to have modest effect on flow field and WSS distribution. ${ }^{21}$ Although the steady flow is not a physiologically relevant assumption, important flow aspects such as secondary flows, and vortical structures are still present under this assumption. ${ }^{6,31}$ Furthermore, the Reynolds number and bend sweep angle effects on the complex flow patterns associated with the S-shaped bend configurations have not been well examined and categorized for steady flows in previous studies.

Under the above-mentioned assumptions, the governing equations including continuity and NavierStokes equations in their integral forms, which are more suitable for the control volume approach used in the present study, are as follows:

$$
\begin{gathered}
\iint \vec{V} \cdot d \vec{A}=0 \\
\int_{\forall} \rho \frac{\partial \vec{V}}{\partial t} d \forall+\iint \rho \vec{V} \vec{V} \cdot d \vec{A} \\
=-\iint p \vec{n} \cdot d \vec{A}+\iint \mu \Delta \vec{V} \cdot d \vec{A},
\end{gathered}
$$

where $\rho, \vec{V}, p$, and $\mu$ are fluid density, velocity vector, pressure, and viscosity, respectively.

A velocity inlet boundary condition with a parabolic profile is considered at the inlet with no slip condition at the walls. As the outlet boundary condition, the axial gradients of all velocity components are set to zero assuming fully develop conditions prevail there. Therefore, a sufficiently long straight section is considered after the bends to make sure that the flow practically recovers its fully developed profile. Consistent with the mass-driven flows, zero pressure gradient is assumed at the inlet and normal to the artery walls, while pressure is set to zero at the outlet corresponding to the fully developed conditions there.

\section{THE NUMERICAL ALGORITHM}

The governing equations are integrated over the corresponding control volumes upon transformation into a generalized nonorthogonal coordinate system. The principal of the numerical method is based upon the calculation of the velocity field from momentum equations using the existing or approximate pressure field. This velocity field does not necessarily satisfy the mass conservation equation, and thus a velocity correction is introduced. A velocity potential is assigned to the velocity correction according to the Hodge decomposition theorem, which states that any vector function can be decomposed into a divergence-free component and the gradient of a scalar potential. This is consistent with the fact that an intermediate velocity field obtained from the momentum equations using an existing pressure field carries the exact vorticity field, and therefore, the velocity correction comes from an irrotational field that can be described with a velocity potential. The continuity equation is then transformed into a Poisson equation for the velocity potential, which is solved with a matrix-free and pre-conditioned version of GMRES. ${ }^{39}$ This method is capable of enhancing the convergence rate of the Poisson solver as compared to the traditional successive-over-relaxation solvers. Finally, the pressure correction is directly related to the velocity correction through the momentum equations. The numerical scheme was originally developed by Chorin, ${ }^{5}$ and improved further by Dwyer et al..$^{8}$ and the present authors. ${ }^{29}$ Further enhancement in convergence rate of the solution procedure is achieved by implementing a pressure correction based on the average velocity defect $\Delta u^{\prime}$ at each cross section of the pipe such that ${ }^{8}$ :

$$
\Delta u^{\prime}=U-U_{i},
$$

where $U$ is the average velocity at a given cross section and $U_{i}$ is the average inlet velocity. Assuming that the local velocity defect is associated with a pressure defect, the following equation can be formed:

$$
\rho \frac{\Delta u^{\prime}}{\Delta t}=-\frac{\partial p^{\prime}}{\partial x}
$$

Thus, the pressure field is updated with the above correction, and then the momentum equations are solved again with the new pressure field. This procedure significantly improves the computational efficiency. The converged steady-state solution is assumed to be achieved, when the absolute relative changes in all flow parameters between two successive iterations are less than $10 \mathrm{E}-05$.

\section{MODEL VALIDATION}

In order to validate the numerical procedure, steady flow in an S-shaped channel similar to the geometry used in the experimental study conducted by Taylor et al..$^{34}$ is considered for the Reynolds number of 790 based on the diameter. The geometry consists of a circular cross section of $48 \mathrm{~mm}$ in diameter, curvature radius of $336 \mathrm{~mm}$, and two successive bends with sweep angles of $22.5^{\circ}$. Straight inflow and outflow sections are 210 and $1820 \mathrm{~mm}$ long, respectively. Uniform velocity profile is imposed at the inlet boundary, while zero gradients and no slip conditions are considered as the outflow and wall boundary 
conditions, respectively. Axial velocity profiles in the curvature plane of the S-shaped bend at three specified cross sections are compared with the corresponding experimental data ${ }^{34}$ in Fig. 2, where reasonable agreements are observed. It should be noted that despite the large differences between the dimensions of the validation case and the models considered here, still both geometries have almost similar curvature ratios.

\section{NORMALIZATION AND GRID SPECIFICATIONS}

In the following sections, the velocity, pressure, WSS, and vorticity defined as the curl of velocity vector field: $\vec{\omega}=\vec{\nabla} \times \vec{V}(\vec{\omega}$ is vorticity and $\vec{V}$ is the

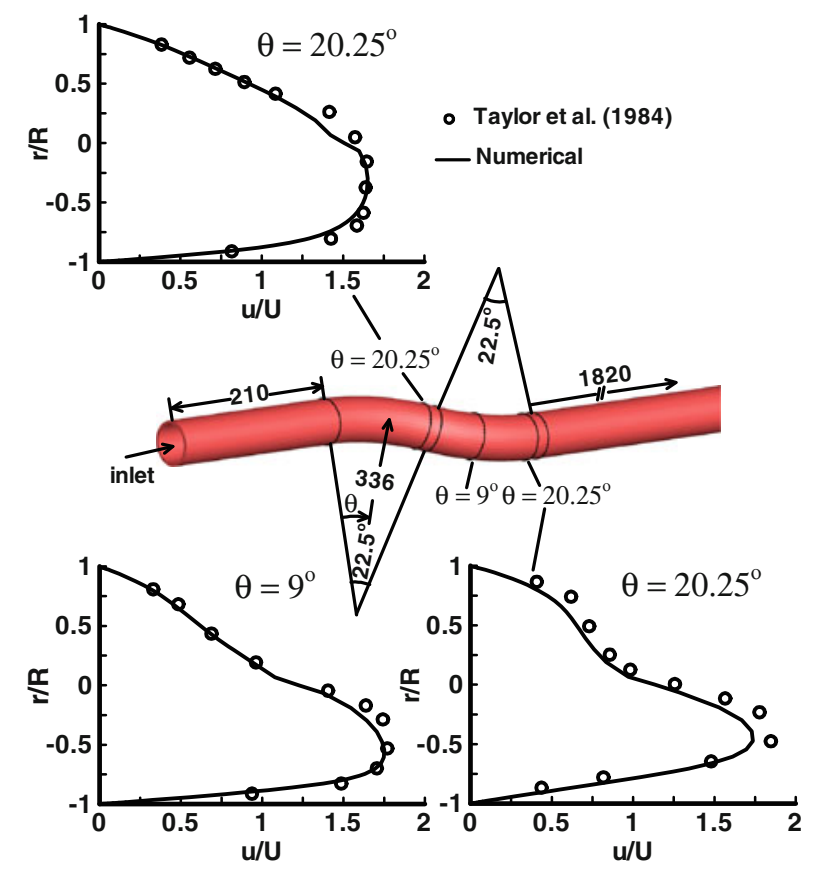

FIGURE 2. Comparison of the axial velocity profiles in the curvature plane of S-shaped bend with $22.5^{\circ}$ sweep angle with the experimental data. ${ }^{34}$ All dimensions in the 3D geometry are in millimeter. velocity vector) are normalized with $U, \frac{1}{2} \rho U^{2}, \rho U^{2}$, and $D / U$, respectively. Here $U$ is the average crosssectional velocity, which is determined from the flow Reynolds number.

Extensive computations have been performed to identify the number of grid points that produces reasonably grid-independent results. It was found the minimum grid points of $42 \times 21 \times 605$ are required in the azimuthal, radial, and axial directions, respectively. Uniform grid spacing is used in the azimuthal direction, while the expansion ratio of 1.15 is employed in the radial direction. In the short straight inflow region and along the bends, grid points are uniformly distributed; however, they expand in the straight section after the bends toward the outlet with the expansion ratio of 1.005 .

\section{MODEL HEMODYNAMICS}

Flow field in S-shaped bends with different sweep angles exhibits some similar features; therefore, the hemodynamics of the $90^{\circ}$ model within the specified range of the Reynolds numbers are presented in Figs. 3-5 to obtain an overall view of the flow behavior. In Fig. 3, the nondimensional axial velocity profiles for Reynolds numbers of 125, 500, and 960 are depicted at 18 specified cross sections in the curvature plane, while Fig. 4 shows the nondimensional axial velocity contours for some selected cross sections. Furthermore, the transverse velocity vectors with axial vorticity contours as the background in the left half and cross-sectional streamlines in the right half of each cross section are shown in Fig. 5. Note that at $\operatorname{Re}=960$, where the flow does not contain a symmetry plane after the second bend turning angle of $75^{\circ}$, the cross-sectional streamlines are shown only for clarity. This asymmetrical feature of the flow will be discussed in more detail later.

Comparing the axial velocity patterns of cross sections located in the first bend with those of a $90^{\circ}$ single bend (not shown here) in Fig. 3 reveals that the

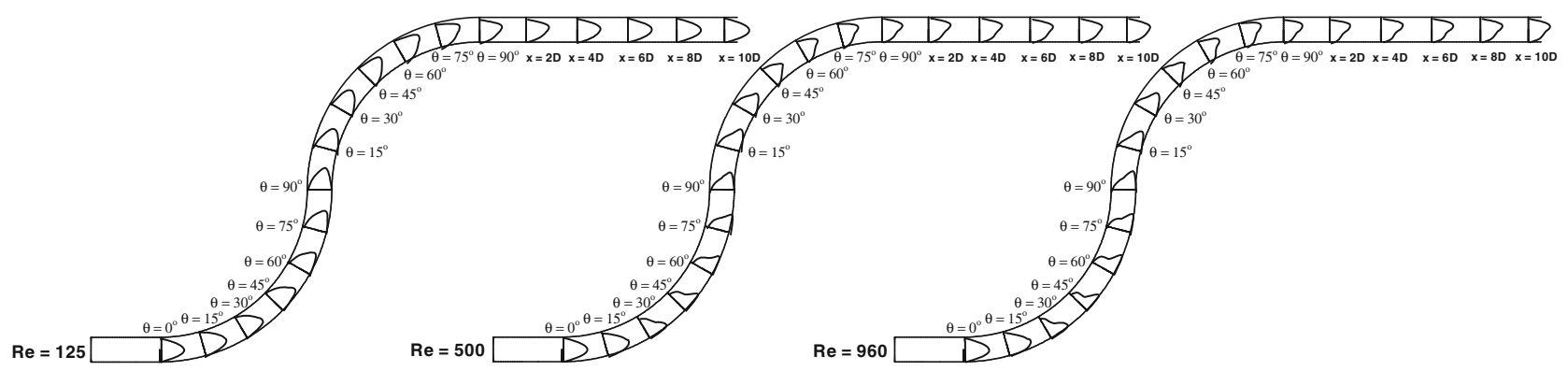

FIGURE 3. Axial variations of nondimensional velocity profiles in the curvature plane of $90^{\circ} \mathrm{S}$-shaped bend for three different Reynolds numbers. 


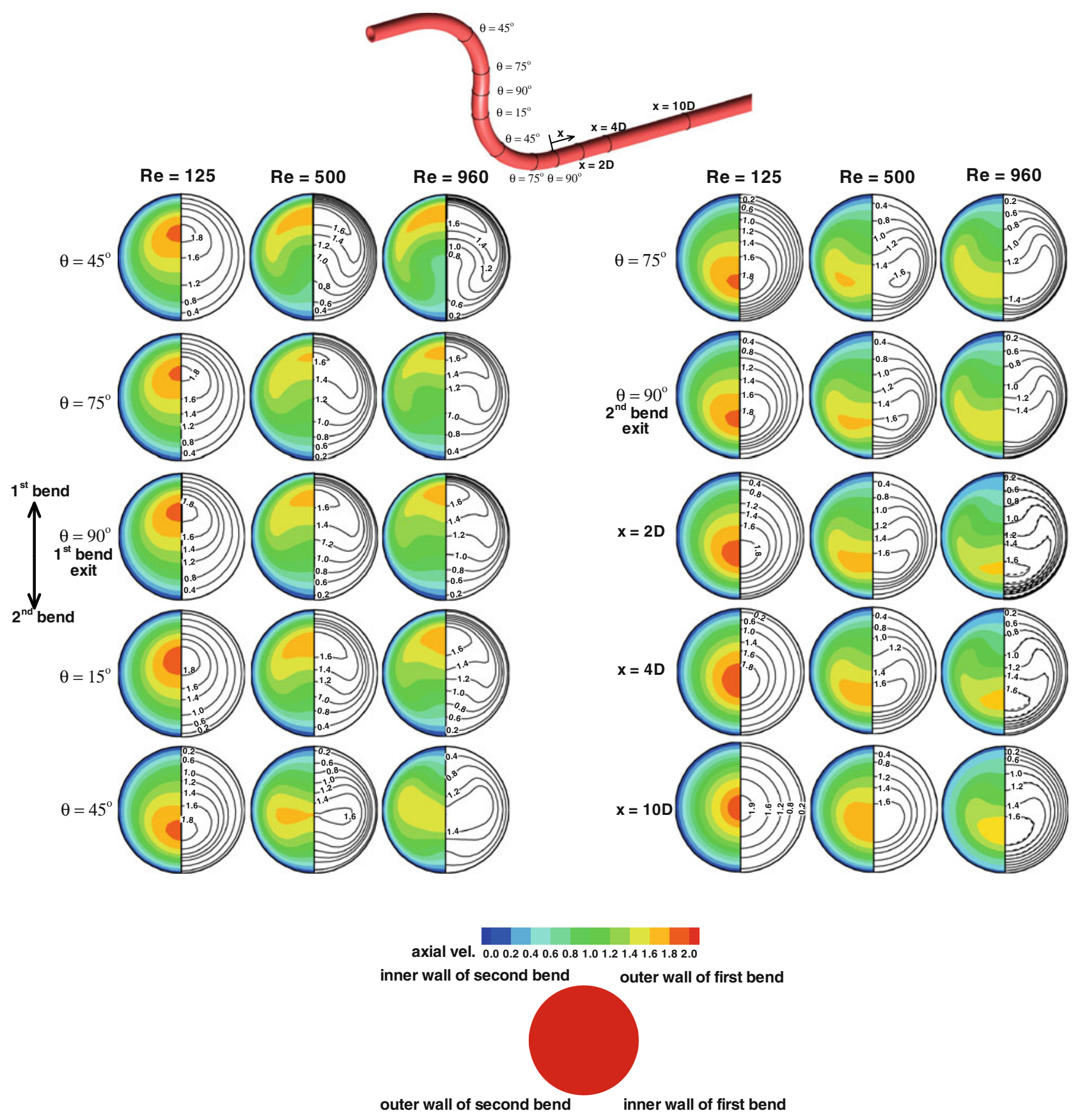

FIGURE 4. Nondimensional axial velocity contours for different Reynolds numbers at some selected cross sections of $90^{\circ}$ $\mathrm{S}$-shaped bend. Dashed lines in the last three cross sections show the symmetric contours, while the solid lines indicate the numerically obtained contours.

flow field in this region is almost independent of the second bend reverse curvature. At the early sections of the first bend, a unidirectional secondary flow forms which results in the slight shift of velocity profiles toward the inner wall of this bend. As the flow moves forward two counter rotating vortices establish (Fig. 5, first bend turning angle of $45^{\circ}$ ) which are the well-known Dean vortices. These vortices cause the flow around the outer wall to move inwardly in the azimuthal direction, while the high-speed particles from the core move toward the outer wall causing a shift in the point of maximum axial velocity accordingly, see Figs. 3 and 4, first bend cross sections. The maximum ratio of transverse velocities to their corresponding axial velocities along the $90^{\circ} \mathrm{S}$-shaped bend for different Reynolds numbers of 125, 500, and 960 are 0.12 , 


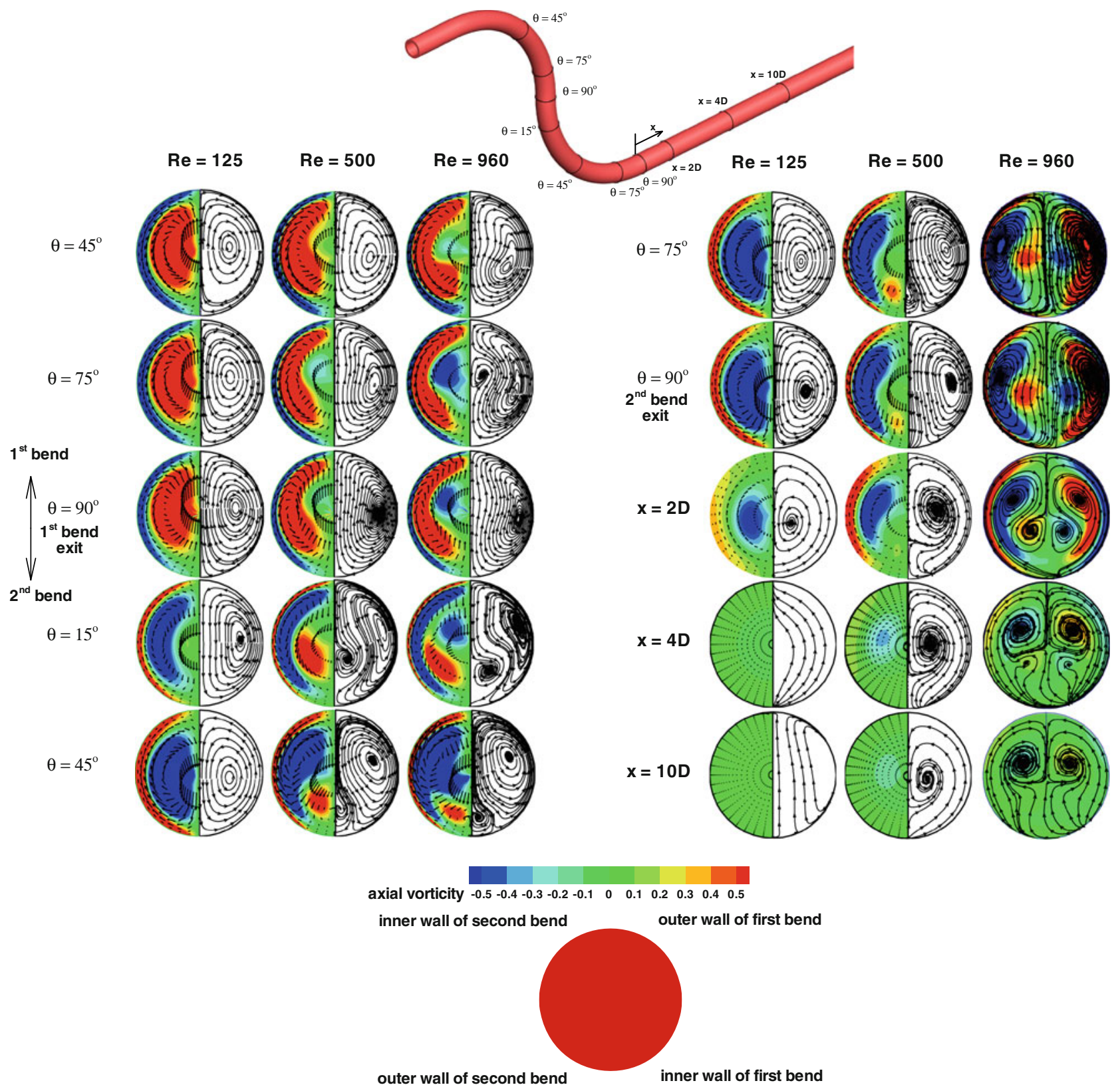

FIGURE 5. Nondimensional transverse velocity vectors with nondimensional axial vorticity as the background in the left half and the secondary flow streamlines in the right half at some selected cross sections of $90^{\circ} \mathrm{S}$-shaped bend for different Reynolds numbers.

0.14 , and 0.16 , respectively. That is, increasing the Reynolds number results in stronger secondary flows contributing to a larger shift of the high velocity region toward the outer wall and introducing a crescent shape into the axial velocity contours (Figs. 3 and 4, first bend cross sections). Furthermore, it is observed that at the Reynolds number of 960 the initial Dean pattern of the first bend transforms into a four-vortex secondary field as seen at the turning angle of $75^{\circ}$. This new pair of vortices rotating with the opposite sense of the earlier pair initiates at the turning angle of about $\theta \approx 55^{\circ}$ and diminishes approximately at $\theta \approx 83^{\circ}$. Similar patterns are observed for sweep angle of $135^{\circ}$ as well.

Further investigation on the formation of fourvortex secondary field in the first bend reveals that this phenomenon is governed by both Reynolds numbers and sweep angles. For example, at $\mathrm{Re}=960$, sweep angles higher than $55^{\circ}$ are required for this pattern to happen. Moreover, it is observed that the new pair preserves its identity for limited turning angles starting from approximately $\theta \approx 55^{\circ}$ and ending at about $\theta \approx 83^{\circ}$ for all sweep angles beyond $55^{\circ}$. 
Secondary flows and axial vorticity patterns in the second bend are strongly dependent upon the Reynolds number and the bend sweep angle. As the flow advances into the second bend, a new pair of vortices consistent with this bend curvature form, which depending on the Reynolds number can partly or fully eliminates the remnants of the first bend vortices. For sufficiently small Reynolds numbers, such as 125 , the newly formed vortices completely annihilate the first bend pair in the early sections of the second bend as shown in Fig. 4, $\mathrm{Re}=125$, second bend turning angle of $15^{\circ}$. However, as the Reynolds number increases the vortices of the first bend become strong enough to persist for a portion or even all the way through the second bend. For example at Reynolds number of 960 in the $90^{\circ}$ bend as shown in Fig. 5, the new vortices of the second bend annihilate the secondary effects of the first bend around the second bend exit. As can be seen in Fig. 4, the annihilation of the secondary effects of the first bend corresponds to the complete shift of the maximum axial velocity points toward the second bend outer wall and the formation of velocity profiles consistent with the bend curvature.

It is worth mentioning that for $\mathrm{Re}=960$ some asymmetric features in the secondary flows around the second bend exit are observed, which maintain along the outflow straight region as shown in Fig. 5. Despite the observable cross-sectional asymmetric flow behavior, the axial velocity contours are slightly affected as shown in the last three cross sections of Fig. 4. For these cross sections located at $x=2 D, 4 D$, and $10 D$, symmetric axial velocity contours are plotted with dashed lines employing the plotting software, while solid lines represent the computationally obtained axial velocity contours. Note that at cross section $x=10 D$ the axial velocity profile is basically symmetric. To the best of our knowledge, symmetrybreaking in S-shaped bends hemodynamic has not been reported in recent studies. Previous numerical studies mainly enforced symmetry condition in their simulations and solved only half of the solution domain to reduce the computational costs, ${ }^{13,15,19}$ which eliminates capturing any asymmetrical features. However, asymmetrical features have been reported in a straight artery with axisymmetric stenosis under pulsatile flow in the form of asymmetric secondary flow patterns and swirling motion in post stenotic region. ${ }^{3,23}$ Furthermore, the occurrence of instabilities and symmetry-breaking phenomenon in the flow through a symmetric sudden expansion beyond a critical range of Reynolds numbers have also been reported. ${ }^{7}$

A numerical computation has been performed to identify whether the present numerical scheme is capable of capturing the asymmetry features of these types of flows reported in literature. For this purpose, a pulsatile flow in axisymmetric stenosis identical to the work of Mallinge and Drikakis ${ }^{23}$ is considered. Our findings compare well with their results for a $75 \%$ axisymmetric stenosis introducing the prescribed perturbations on the inlet velocity profile. Present results regarding the asymmetry flows in S-shaped bends indicate that this phenomenon is related to the simultaneous effects of geometry and Reynolds number, which will be discussed later.

Leaving the second bend, the secondary flows weaken dramatically, while the axial velocity profile recovers its parabolic shape. However, the higher the Reynolds number the longer it takes for the axial velocity profiles to recover.

\section{SWEEP ANGLE AND REYNOLDS NUMBER EFFECTS}

As mentioned earlier, flow field in S-shaped bends is affected by their geometrical configurations and Reynolds number. In general, flow in the first bend forms a Dean vortex consistent with its curvature. However, in the second bend where the curvature of the bend is reversed a new pair of vortices forms, which rotates in the opposite sense of the first bend vortices. Depending upon the Reynolds number and the sweep angle different scenarios may happen in the second bend. At low enough Reynolds numbers for all sweep angles, flow in the second bend behaves consistent with its curvature in the sense that the vortices of the second bend forming close to the wall overcome the core vortices of the first bend in the early sections of the second bend and occupy the entire cross section. However, at higher Reynolds numbers but low sweep angles the vortices of the second bend are not strong enough to overcome the powerful vortices of the first bend and therefore, maximum axial velocity occurs in a region which is not consistent with the second bend curvature and a four-vortex structure can be observed throughout the second bend and even at some portion of the outlet straight section. However, by increasing the sweep angle the vortices of the second bend experience the centripetal forces for longer distance and become more energetic such that they may annihilate the first bend vortices before the flow passes through the second bend exit.

To investigate the geometrical effects, in Fig. 6 the nondimensional axial velocity profiles are plotted at specified cross sections along the curvature plane of bends with different sweep angles at Reynolds number of 960 . Clearly, the axial velocity profiles in the second bend of the $45^{\circ}$ model are inconsistent with the bend curvature and remain shifted toward its inner wall. To 


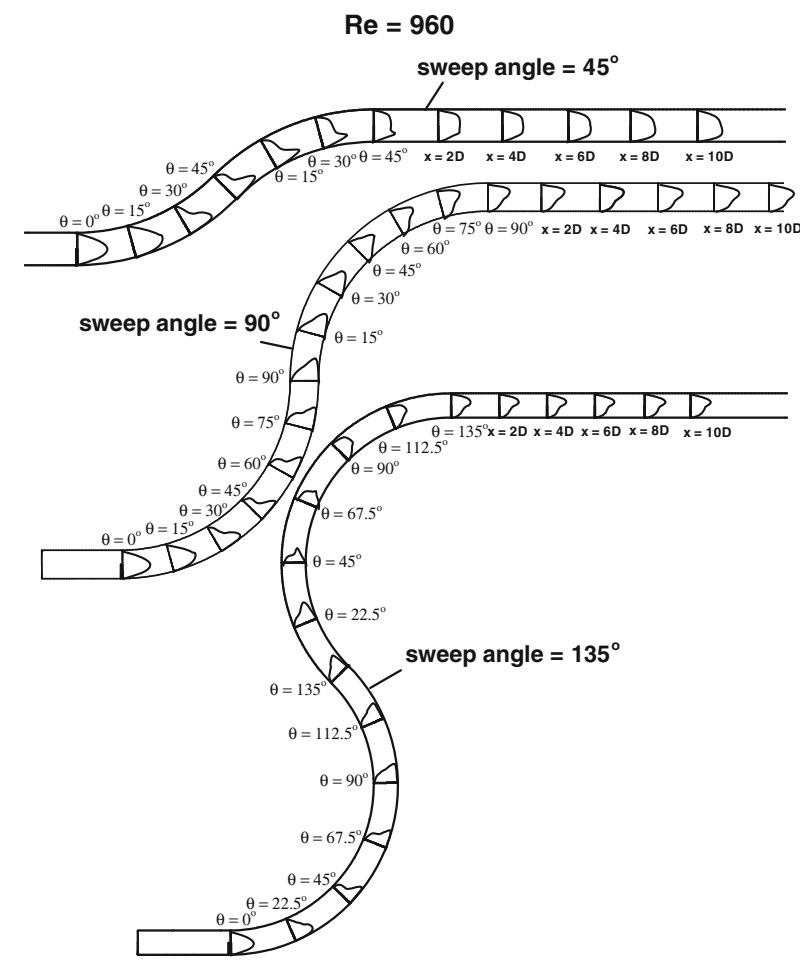

FIGURE 6. Nondimensional axial velocity profile variations in the curvature plane of S-shaped bends with different sweep angles for $\mathrm{Re}=960$.

bring more clarity to this phenomenon, the transverse streamlines for different sweep angles are plotted at some selected cross sections in Fig. 7 for the same Reynolds number. The bend exit cross section of the $45^{\circ}$ model shows the core vortices remnant of the first bend wrapped by the oppositely rotating vortices of the second bend. However, the cross-sectional streamlines of the other models at the bend exit show almost no traces of the first bend vortices. It is noteworthy that secondary flow asymmetry can be observed in all three models; however, the $90^{\circ}$ model shows the largest asymmetric features by introducing slight asymmetry to the main flow profiles as well as shown in Fig. 4.

\section{SYMMETRY-BREAKING PHENOMENON}

Reynolds number and geometry are believed to play important roles in introducing asymmetrical features into the flow field of symmetric geometries such as sudden expansions and stenosed arteries. ${ }^{3,7,23}$ It is shown that flows in symmetric geometries with symmetric flow conditions may exhibit asymmetrical behaviors within a critical range of Reynolds numbers. ${ }^{18,23,24}$ These asymmetrical patterns are usually unsteady, however, in some cases such as flow over a

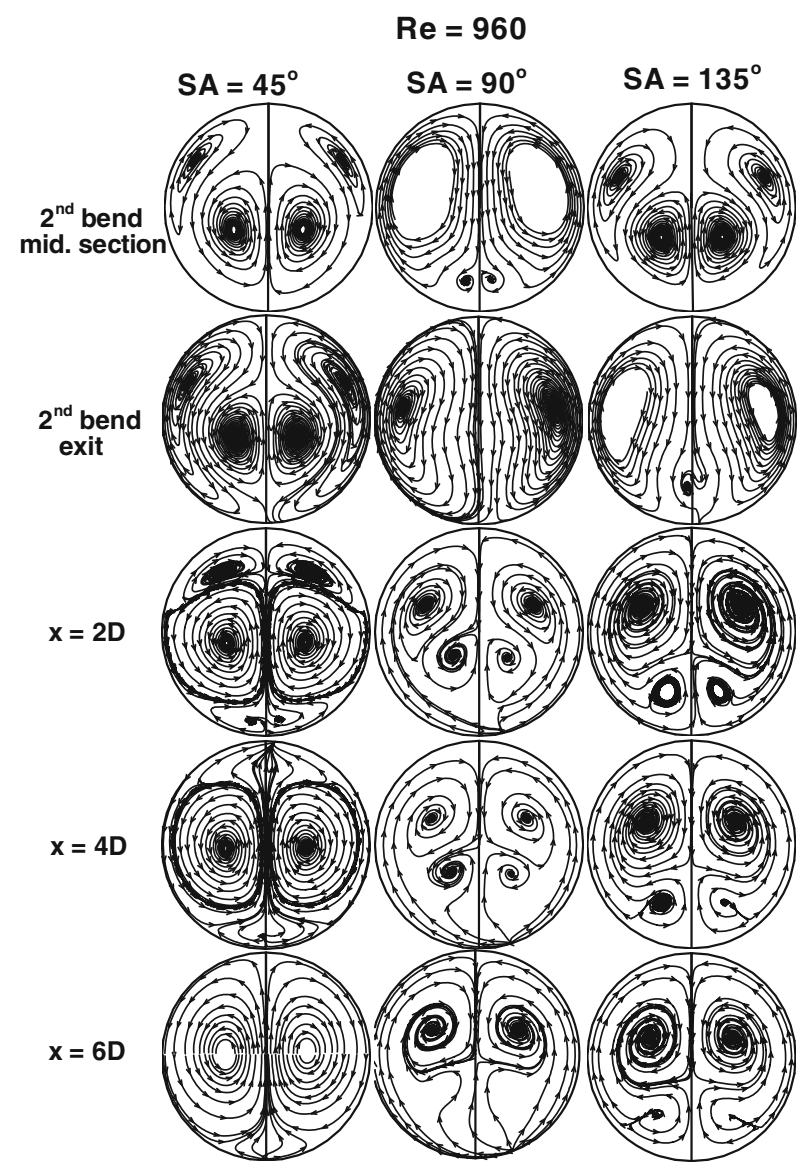

FIGURE 7. Secondary flow streamlines of S-shaped bends with different sweep angles at some selected cross sections for $\operatorname{Re}=960$.

solid sphere a steady attached asymmetrical wake structure is observed in a specific range of Reynolds numbers, which then becomes unsteady at higher Reynolds numbers. ${ }^{18,24}$ Therefore, in order to understand the effect of Reynolds number on the symmetry-breaking phenomenon in S-shaped bends, a numerical study at a high Reynolds number of 1920 has been conducted for all the three sweep angles. It should be noted that this Re number does not occur in femoral arteries and is only considered here to explore the effect of Reynolds number on the symmetry-breaking phenomenon. In Fig. 8, the axial velocity contours (at the top) and the transverse streamlines (at the bottom) at some selected cross sections of S-shaped bends with different sweep angles are shown. The cross-sectional streamlines at the second bend exit of $45^{\circ}$ and $135^{\circ}$ bends show almost no traces of asymmetrical features, while at the same cross section the velocity patterns of bend with $90^{\circ}$ sweep angle exhibit asymmetrical behavior. However, as the flow advances into the outflow region slight asymmetrical features are also observed in the $45^{\circ}$ and $135^{\circ}$ bends secondary flows. 


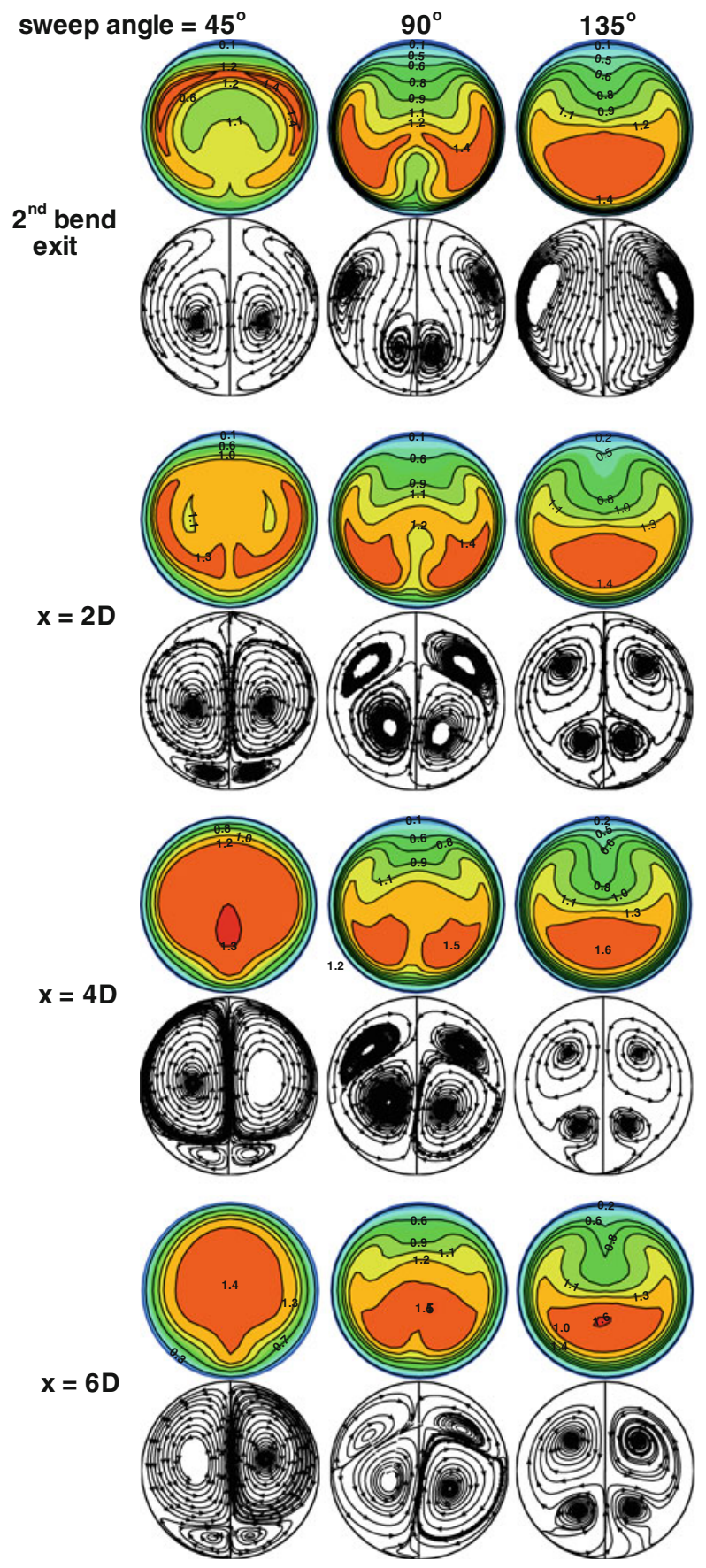

$\mathrm{Re}=1920$

sweep angle $=45^{\circ}$

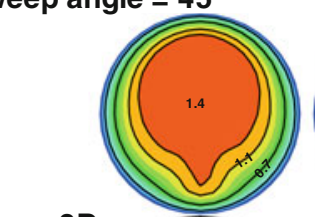

$\mathrm{X}=\mathbf{8 D}$
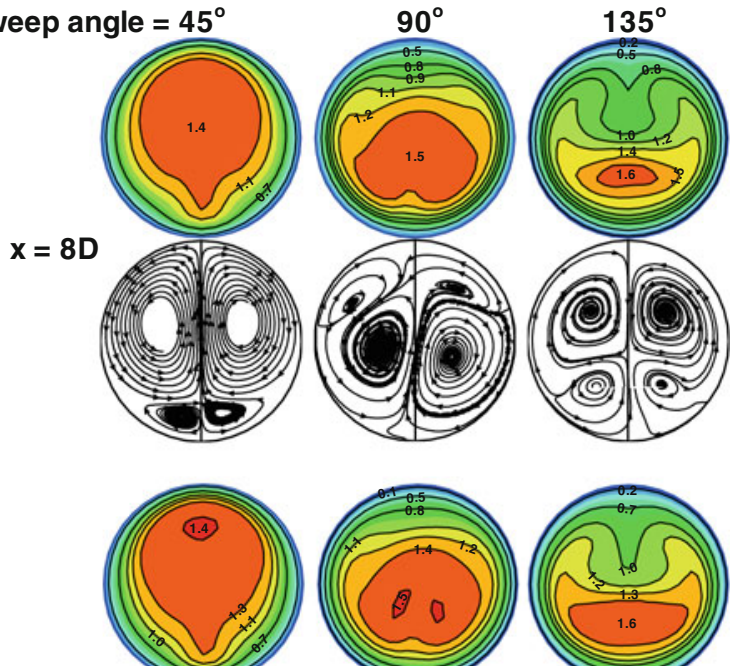

$x=10 D$
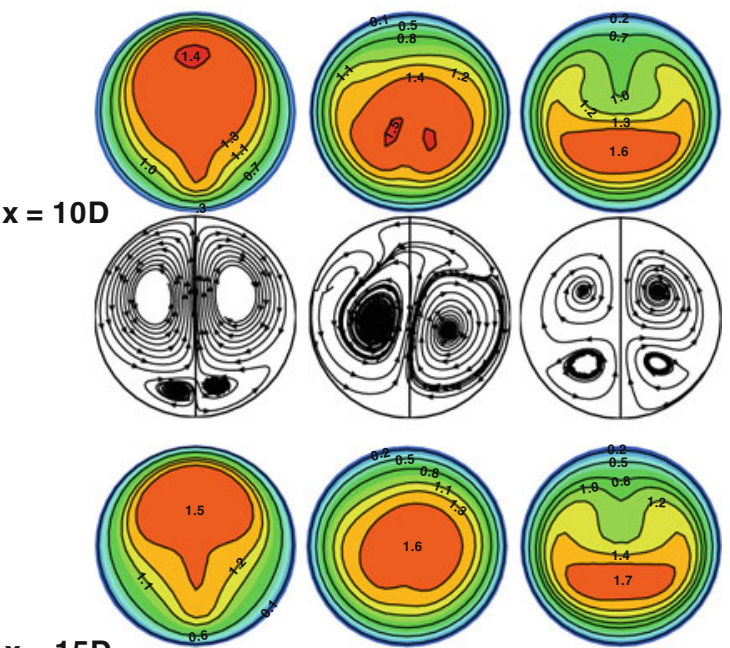

$x=15 D$
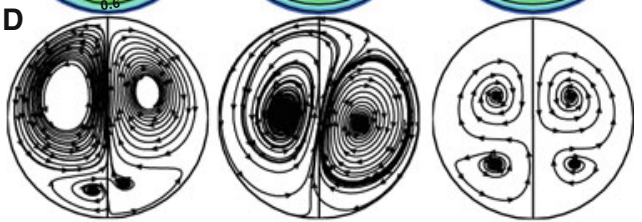

outer wall of second bend

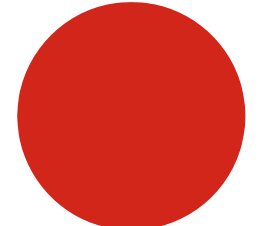

inner wall of second bend

FIGURE 8. Axial velocity contours (at the top) along with the corresponding cross-sectional streamlines (at the bottom) for $\operatorname{Re}=1920$ at some selected cross sections of S-shaped bend with different sweep angles.

Despite these asymmetrical secondary flows, the axial velocity contours almost remain symmetric. The $90^{\circ}$ model shows the most asymmetrical secondary patterns where a pair of almost Dean vortex oriented to the right-hand side gradually forms, which is directly associated with the shift of the axial velocity profile toward this side of cross sections as shown in Fig. 8, $x=15 D$. According to these observations, at higher Reynolds numbers the stream wise asymmetrical features are shown to become more apparent in the flow field of S-shaped bends with $90^{\circ}$ sweep angle, while the main flow of $45^{\circ}$ and $135^{\circ}$ models remain almost symmetric, even at such a high Reynolds number. 


\section{FLOW SEPARATION IN S-SHAPED BENDS}

Flow separation is one of the significant phenomena in the study of blood hemodynamics which is broadly believed to be associated with several arterial wall diseases. $^{9,22}$ Therefore, recognition of the regions prone to flow separation and the parameters that govern the initiation of this phenomenon is of practical importance.

Flow separation is directly related to the existence of adverse pressure gradient. For an S-shaped bend adverse pressure gradient always occurs at the early sections of the first bend outer wall, while it may occur at the transition of the two bends along the second bend outer wall depending upon the Reynolds number, sweep angle, and curvature ratio. For a given curvature ratio as is the case of the present study and the Reynolds numbers considered here no separation occurs at the outer wall of the first bend, despite the existence of the adverse pressure gradients there, which indicates that the fluid momentum in this region is high enough to overcome the opposing adverse pressure gradient.

Further investigations on Reynolds number and sweep angle effects on flow separation show that separation along the second bend outer wall can only occur in gentle bends with low sweep angles of $20^{\circ}$ up to $30^{\circ}$, and high Reynolds numbers such as $\operatorname{Re}=1920$. Present computations for gentle bends with different Re show no trace of separation for Reynolds numbers smaller than 1500, while, no further attempts have
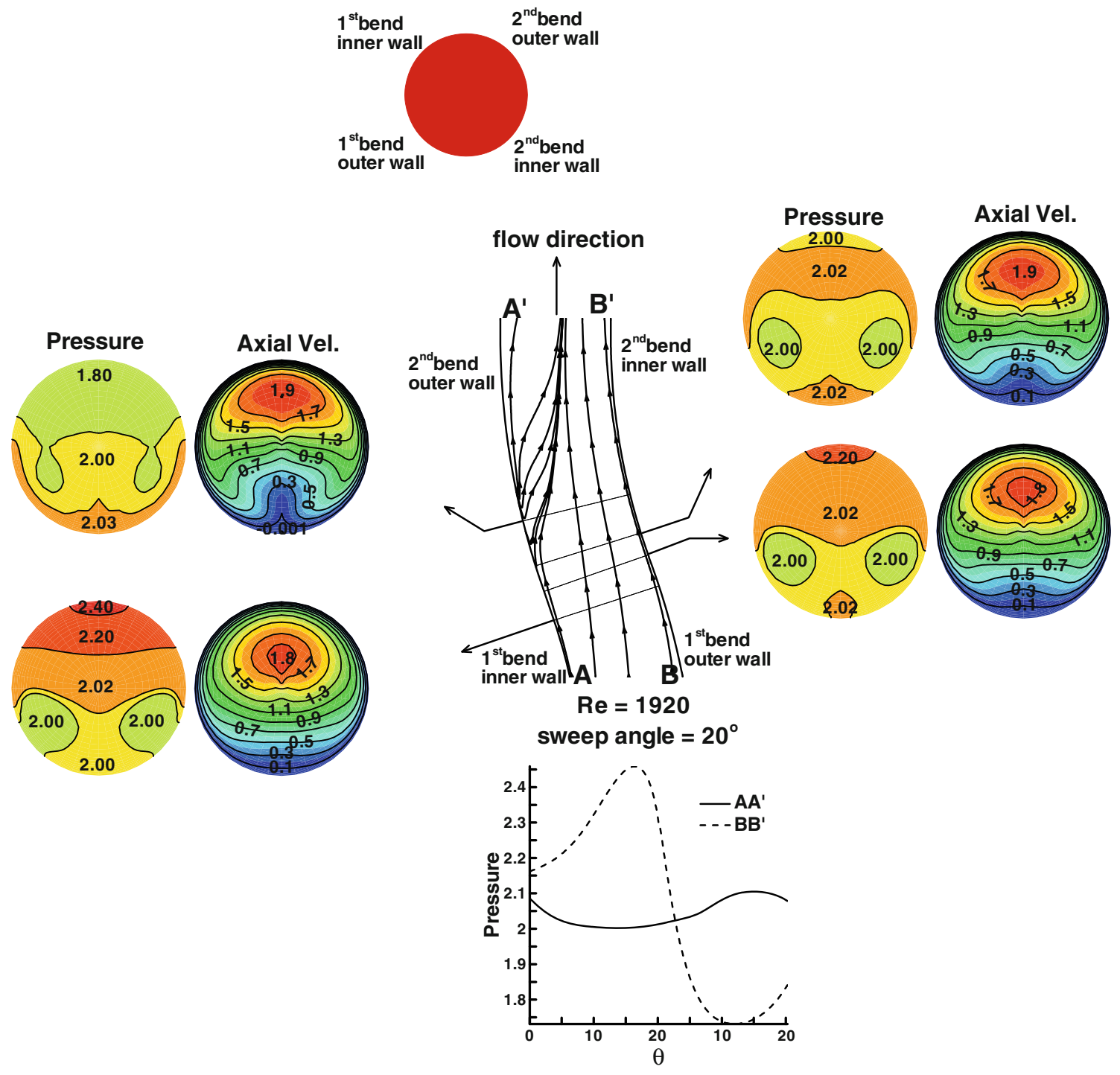

FIGURE 9. Flow streamlines in the symmetry plane of $20^{\circ} \mathrm{S}$-shaped bend along with the contours of nondimensional pressure and axial velocity fields at some selected sections for Reynolds number of 1920. 
been made to identify the exact Re that initiates separation for gentle bends. This is, however, in contrast with the results of Hoogstraten et al. ${ }^{15}$ indicating that flow separation can occur in a $35^{\circ} \mathrm{S}$-shaped bend at Reynolds number of 960.

To study the separation features in gentle bends the flow streamlines in the symmetry plane of a $20^{\circ}$ S-shaped bend at $\mathrm{Re}=1920$ are shown in Fig. 9. This figure presents the cross-sectional nondimensional pressure and axial velocity contours at four cross sections around the interface of the two bends, in addition to the surface pressure variations along the $\mathrm{AA}^{\prime}$ and $\mathrm{BB}^{\prime}$ lines. It is observed that for these flow conditions separation from arterial wall occurs along the first bend inner and second bend outer wall in the bends transitional region. Despite the stronger adverse pressure gradients that exist along the $\mathrm{BB}^{\prime}$ line as compared to $\mathrm{AA}^{\prime}$ line, flow remains attached to the wall in this region since fluid particles are energetic enough to climb the corresponding pressure hill there without separating from the wall. Effects of bend sweep angle on separation are also studied. It is observed that the separation length and strength decrease as the bends sweep angle increases.

\section{SUMMARY AND CONCLUSIONS}

Steady flow patterns in S-shaped bends with sweep angles of $45^{\circ}, 90^{\circ}$, and $135^{\circ}$ for a fixed curvature ratio of 13 are investigated numerically. Results show that the flow fields in these geometries are generally dependent on the Reynolds number and sweep angles which can be categorized into three different groups. Low Reynolds numbers flows, which are almost independent of sweep angles. Dean vortex pattern in both bends are consistent with the bend curvature except for the early sections of the second bend. Low Re flows are practically geometry-dominated flows. At higher Reynolds numbers with lower sweep angles, the secondary vortices of the first bend apparently remain in the core of the second bend wrapped by vortices of this bend, and therefore, each cross section in the second bend contains two pairs of vortices. Axial velocity profiles in the second bend carry the first bend features all the way through the second bend. At higher sweep angles, the secondary vortices of the second bend become strong enough to overcome the effects of the first bend on the axial velocity profiles shifting the maximum velocity in direction consistent with the second bend curvature. It is worth mentioning that for cases where two pairs of vortices exist in the second bend cross sections, the core vortices related to the first bend become dominant along the outflow region.
Separation along the outer wall of second bend is also detected for gentle bends with sweep angles between $20^{\circ}$ up to $30^{\circ}$ and Reynolds numbers as high as 1920. For such flow conditions, flow is separated due to the adverse pressure gradient along the first bend inner wall and the second bend outer wall. Moreover, the separation length at a given Reynolds number shortens as the bend sweep angle increases.

An interesting feature associated with high Reynolds numbers flows is the symmetry-breaking phenomenon in the secondary field of the S-shaped bends at any sweep angles. Present results indicate that the asymmetrical axial velocity profiles are more pronounced for sweep angle of $90^{\circ}$.

It should be mentioned that the potential limitations of the current study are the following assumptions: (a) Newtonian fluid, (b) rigid wall, and (c) steady flow. Although these assumptions may lead to some deviations from the realistic physiological conditions, however, as mentioned earlier these assumptions can be reasonably applied for the blood flow investigation without loss of generality.

\section{REFERENCES}

${ }^{1}$ Back, L. H., T. K. Liem, E. Y. Kwack, and D. W. Crawford. Flow measurements in a highly curved atherosclerotic coronary artery cast of man. J. Biomech. Eng. 114:232-240, 1992.

${ }^{2}$ Banerjee, R. K., Y. I. Cho, and L. H. Back. Numerical studies of three-dimensional arterial flows in reverse curvature geometry: part I-peak flow. J. Biomech. Eng. 115:316-326, 1993.

${ }^{3}$ Blackburn, H. M., S. J. Sherwin, and D. Barkley. Convective instability and transient growth in steady and pulsatile stenotic flows. J. Fluid Mech. 607:267-277, 2008.

${ }^{4}$ Channon, K. M. The endothelium and the pathogenesis of atherosclerosis. Medicine 34:173-177, 2006.

${ }^{5}$ Chorin, A. J. Numerical solution of the Navier-Stokes equations. Math. Comp. 22:745-762, 1968.

${ }^{6}$ Doorly, D., and S. Sherwin. Chapter in Cardiovascular Mathematics, edited by L. Formaggia, A. Quarteroni, and A. Veneziani. New York: Springer, 2009.

${ }^{7}$ Drikakis, D., F. Grinstein, and D. Youngs. On the computation of instabilities and symmetry-breaking in fluid mechanics. Progr. Aerospace Sci. 41:609-641, 2005.

${ }^{8}$ Dwyer, H. A., A. Y. Cheer, T. Rutangnira, and N. Shahcheraghi. Calculation of unsteady flows in curved pipes. J. Fluids Eng. 123:869-877, 2001.

${ }^{9}$ Ethier, C. R., S. Prakash, D. A. Steinman, R. L. Leask, G. G. Couch, and M. Ojha. Steady flow separation patterns in a 45 degree junction. J. Fluid Mech. 411:1-38, 2000.

${ }^{10}$ Fan, Y., Z. Xu, W. Jiang, X. Deng, K. Wang, and A. Sun. An S-type bypass can improve the hemodynamics in the bypassed arteries and suppress intimal hyperplasia along the host artery floor. J. Biomech. 41:2498-2505, 2008. 
${ }^{11}$ Gijsen, F. J. H., F. N. Van De Vosse, and J. D. Janssen. The influence of the non-Newtonian properties of blood flow in a carotid bifurcation model. J. Biomech. 32:601608, 1999.

${ }^{12}$ Giannoglou, G. D., J. V. Soulis, T. M. Farmakis, G. A. Giannakoulas, G. E. Parcharidis, and G. E. Louridas. Wall pressure gradient in normal left coronary artery tree. Med. Eng. Phys. 27:455-464, 2005.

${ }^{13}$ Grigioni, M., K. Daniele, U. Morbiduuci, C. D. Gaudio, G. D. Avenio, A. Balducci, et al. A mathematical description of blood spiral flow in vessels: application to a numerical study of flow in arterial bending. J. Biomech. 38:1375-1386, 2005.

${ }^{14}$ Hong, J., L. Wei, C. Fu, and W. Tan. Blood flow and macromolecular transport in complex blood vessels. Clin. Biomech. 23:125-129, 2008.

${ }^{15}$ Hoogstraten, H. W., J. G. Kootstra, B. Hillen, J. K. B. Krijger, and P. J. W. Wensing. Numerical simulation of blood flow in an artery with two successive bends. J. Biomech. 29:1075-1083, 1996.

${ }^{16}$ Johnston, P. R., and B. M. Johnston. Blood flow in S-shaped in-plane and out-of-plane coronary arteries. ANZIAM 49:341-358, 2008.

${ }^{17}$ Johnston, B. M., P. R. Johnston, S. Corney, and D. Kilpatrick. Non-Newtonian blood flow in human right coronary arteries: Steady state simulations. J. Biomech. 37:709-720, 2004.

${ }^{18}$ Johonson, T. A., and V. C. Patel. Flow past a sphere up to a Reynolds number of 300. J. Fluid Mech. 378:19-70, 1999.

${ }^{19} \mathrm{Liu}, \mathrm{B}$. The influence of stenosis on the downstream flow pattern in curved arteries. Med. Eng. Phys. 29:868-876, 2007.

${ }^{20}$ Lee, K. E., K. H. Parker, C. G. Caro, and S. J. Sherwin. The spectral/hp element modeling of the steady flow in non-planar double bends. Int. J. Numer. Meth. Fluids. 57:519-529, 2008.

${ }^{21}$ Lee, K. W., and X. Y. Xu. Modeling of flow and wall behavior in a mildly stenosed tube. Med. Eng. Phys. 24:575-586, 2002.

${ }^{22}$ Leondes, C. Hemodynamics simulations and optimal computer-aided designs of branching blood. In: Biomechanical Systems: Techniques and Applications, edited by C. Leondes. Boca Raton: CRC Press, 2001, pp. 1-69.

${ }^{23}$ Mallinge, F., and D. Drikakis. Instability in three-dimensional, unsteady, stenotic flows. Int. J. Heat Fluid Flow 23:657-663, 2002.

${ }^{24}$ Niazmand, H., and M. Renksizbulut. Flow past a spinning sphere with surface blowing and heat transfer. J. Fluids Eng. 127:163-171, 2005.

${ }^{25}$ Nosovitsky, V. A., O. J. Ilegusi, J. Jiang, P. H. Stone, and C. L. Feldman. Effects of curvature and stenosis like narrowing on wall shear stress in a coronary artery model with phasic flow. Comput. Biomed. Res. 30:61-82, 1997.
${ }^{26}$ Olsson, A. G. Atherosclerosis: Biology and Clinical Science. London: Churchill Livingstone, 1987.

${ }^{27}$ Perktold, K. R., M. Nerem, and R. O. Peter. A numerical calculation of flow in curved tube model of left main coronary artery. J. Biomech. 24:175-189, 1991.

${ }^{28}$ Rappitsch, G., K. Perktold, and E. Penkopf. Numerical modeling of shear-dependent mass transfer in large arteries. Int. J. Numer. Meth. Fluids 25:847-857, 1997.

${ }^{29}$ Renksizbulut, M., H. Niazmand, and G. Tercan. Slip-flow and heat transfer in rectangular microchannels with constant wall temperature. Int. J. Therm. Sci. 45:870-881, 2006.

${ }^{30}$ Shahcheraghi, N., H. A. Dwyer, A. Y. Cheer, A. I. Barakat, and T. Rutaganira. Unsteady and three-dimensional simulation of blood flow in the human aortic arch. J. Biomech. Eng. 124:378-387, 2002.

${ }^{31}$ Sherwin, S. J., and D. J. Doorly. Flow dynamics within model distal arterial bypass grafts. In: Vascular Grafts: Experiment and Modeling, edited by A. Tura. Southampton and Boston: WIT, 2003, pp. 327-374.

${ }^{32}$ Soulis, J. V., G. D. Giannoglou, G. E. Parcharidis, and G. E. Louridas. Flow parameters in left coronary artery tree. Implication to atherogenesis. Comput. Biol. Med. 37:628-636, 2007.

${ }^{33}$ Soulis, J. V., T. M. Farmaki, G. D. Giannoglou, and G. E. Louridas. Wall shear stress in normal left coronary artery tree. J. Biomech. 39:742-749, 2006.

${ }^{34}$ Taylor, A. M. K. P., J. H. Whitelaw, and M. Yianneskis. Developing flow in S-shaped ducts 2: circular cross-section duct. NASA Contractor Report 3759, 1984.

${ }^{35}$ Towne, C. E. Computations of viscous flow in curved ducts and comparison with experimental data. In: AIAA Paper AIAA-84-0531, 22nd Aerospace Sciences Meeting, Reno, NV, Jan. 9-12, 1984.

${ }^{36}$ Tsai, S. F., and W. H. Sheu. Finite element analysis of three-dimensional vortical flow structure and topology inside a carotid bifurcation model. Int. J. Comput. Fluid D 21:29-36, 2007.

${ }^{37}$ Van De Vosse, F. N., A. A. V. Steenhoven, A. Segal, and J. D. Janssen. A finite element analysis of the steady laminar entrance flow in a 90 curved tube. Int. J. Numer. Meth. Fluids 9:275-287, 1989.

${ }^{38}$ Wada, S., and T. Karino. Theoretical prediction of lowdensity lipoproteins concentration at the luminal surface of an artery with a multiple bend. Ann. Biomed. Eng. 30:778791,2002

${ }^{39}$ Wigton, L. B., N. J. Yu, and D. P. Young. GMRES acceleration of computational fluid dynamics codes. In: AIAA Computational Fluid Dynamic Meeting, Cinn., Ohio, 1985, pp. 67-74.

${ }^{40}$ Yao, H., K. C. Ang, J. H. Yeo, and E. K. W. Sim. Computational modeling of blood flow through curved stenosed arteries. J. Med. Eng. Technol. 24:163-168, 2000. 